\title{
The Development of a Resolution-Independent Tropical Cyclone Detection Scheme for High-Resolution Climate Model Simulations
}

\author{
Akihiko MURATA \\ Meteorological Research Institute, Tsukuba, Japan \\ Shun-ichi I. WATANABE \\ Office of Global Environment and Climate Research Promotion, \\ Japan Meteorological Business Support Center, Tsukuba, Japan \\ Hidetaka SASAKI, Hiroaki KAWASE, and Masaya NOSAKA \\ Meteorological Research Institute, Tsukuba, Japan \\ (Manuscript received 28 July 2018, in final form 15 January 2019)
}

\begin{abstract}
A novel method for detecting tropical cyclones in high-resolution climate model simulations is proposed herein and subjected to examination. The proposed method utilizes a two-dimensional scatterplot based on two quantities that represent the radial gradient and the tangential asymmetry of mid-to upper-level thickness around a simulated vortex. A comparison between the modeled and observed tropical cyclones using the nonhydrostatic regional climate model (NHRCM) with 20-km grid spacing under reanalysis-driven boundary conditions for one year revealed that no cyclones were missed and there was only one false alarm over a part of the western North Pacific near Japan. The simulated vortices were classified into two categories: tropical cyclones and extratropical cyclones. These two groups, having specific features, were also found in the results using present-day climate datasets, indicating that the tropical cyclones were reasonably distinguished from extratropical cyclones, although a one-by-one comparison could not, in principle, be conducted. Comparison of the results obtained from datasets with $5 \mathrm{~km}$ and $20 \mathrm{~km}$ grid spacing demonstrated that the detection scheme was only weakly dependent on the horizontal resolution. This dependence was further reduced by using the radial gradient over the outer radii instead of near the center of the vortex. The resolution-independent feature in this method is due to a procedure in which the tangential asymmetry of mid-to upper-level thickness is utilized instead of the relative vorticity at 850 $\mathrm{hPa}$, often used in conventional schemes. This procedure allows the method to identify tropical cyclones without the need to determine a grid-dependent threshold. The method proposed here provides a useful tool for detecting tropical cyclones in high-resolution climate simulations.
\end{abstract}

Keywords tropical cyclone; detection scheme; tracking; regional climate model

Citation Murata, A., S. I. Watanabe, H. Sasaki, H. Kawase, and M. Nosaka, 2019: The development of a resolution-independent tropical cyclone detection scheme for high-resolution climate model simulations. J. Meteor. Soc. Japan, 97, 519-531, doi:10.2151/jmsj.2019-035.

Corresponding author: Akihiko Murata, Meteorological Research Institute, 1-1 Nagamine, Tsukuba, Ibaraki 305-0052, Japan

E-mail: amurata@mri-jma.go.jp

J-stage Advance Published Date: 4 February 2019

(C) The Author(s) 2019. This is an open access article published by the Meteorological Society of Japan under a Creative Commons Attribution 4.0 International (CC BY 4.0) license (https://creativecommons.org/licenses/by/4.0). 


\section{Introduction}

Based on climate model projections, precipitation extremes will increase in warm climates. According to the fifth assessment report of the United Nations Intergovernmental Panel on Climate Change (IPCC AR5), "extreme precipitation events over most of the midlatitude land masses and over wet tropical regions will very likely become more intense and more frequent by the end of this century, as the global mean surface temperature increases" (IPCC 2013). This indicates that the intensity of extreme precipitation, such as the annual daily maximum precipitation, tends to increase more significantly compared with average precipitation, such as the annual total precipitation and the monthly total precipitation.

An extreme precipitation event is often associated with a tropical cyclone (e.g., Nakano et al. 2010; Kanada et al. 2017). Therefore, examining future changes in the precipitation amounts accompanying tropical cyclones is a crucial issue for estimating changes in precipitation extremes. It is thus necessary to classify precipitation, simulated by climate models, into two categories: precipitation associated with tropical cyclones and others. To do so, a scheme for detecting tropical cyclones is required. In particular, a scheme suitable for output data from a high-resolution climate model, such as a regional climate model (RCM) and a high-resolution general circulation model (GCM; e.g., Miyamoto et al. 2013), is desirable because our main target is regional precipitation associated with tropical cyclones around Japan in the present and future climates.

Many schemes have been proposed for the detection of tropical cyclones in a gridded dataset produced by climate model simulations. Ullrich and Zarzycki (2017) reviewed schemes for tropical cyclone detection (in Appendix B of their paper). They listed many published schemes along with short descriptions. All of the schemes utilized some kind of threshold value to detect tropical cyclones. For example, surface wind speed is often used and a threshold is set to identify tropical cyclones. An appropriate value of the threshold was examined by Walsh et al. (2007) for different horizontal resolutions. They found that the threshold wind speed varied linearly with resolution. It should be noted that the thresholds are sometimes adjusted so that simulated tropical cyclones show a reasonable number (e.g., Oouchi et al. 2006).

In many schemes, the $850 \mathrm{hPa}$ relative vorticity near the vortex center needs to exceed a threshold for identifying the vortex as a tropical cyclone. For exam- ple, in Bengtsson et al. (1982), one of the criteria for identifying tropical cyclones from a forecast model output with about a $200 \mathrm{~km}$ resolution was that the $850 \mathrm{hPa}$ relative vorticity maxima were greater than 7 $\times 10^{-5} \mathrm{~s}^{-1}$ in a $7.5^{\circ} \times 7.5^{\circ}$ area. Sugi et al. (2002) used an $850 \mathrm{hPa}$ relative vorticity threshold of $7 \times 10^{-5}$ $\mathrm{s}^{-1}$ for a T106 (about $125 \mathrm{~km}$ ) dataset derived from a GCM. In contrast, the threshold value was $3.5 \times 10^{-5}$ $\mathrm{s}^{-1}$ for a $20 \mathrm{~km}$ dataset in Oouchi et al. (2006). On the other hand, Murakami and Sugi (2010) employed resolution-dependent thresholds of $850 \mathrm{hPa}$ relative vorticity for outputs from GCMs with horizontal resolutions between $20 \mathrm{~km}$ and $180 \mathrm{~km}$. As for RCM datasets, one of the criteria for identifying tropical cyclones from a $60 \mathrm{~km}$ grid RCM dataset in Au-Yeung and Chan (2012) was that the local maximum relative vorticity at $850 \mathrm{hPa}$ was greater than or equal to $4.5 \times$ $10^{-4} \mathrm{~s}^{-1}$. Huang and Chan (2014) also used this kind of criterion, although the threshold value was different.

Temperature anomalies in the upper troposphere have also been used to identify tropical cyclones in gridded datasets. This is based on the warm core structure of tropical cyclones. On a practical level, in many schemes, temperature anomalies in upper troposphere near the vortex center need to be larger than a certain threshold. For example, a criterion adopted in Sugi et al. (2002) was that temperature differences from a surrounding area averaged at four pressure levels (i.e., $850 \mathrm{hPa}, 700 \mathrm{hPa}, 500 \mathrm{hPa}$, and $300 \mathrm{hPa}$ ) was greater than 3 K. Oouchi et al. (2006) and Murakami and Sugi (2010) also used this criterion, but at only three levels (i.e., $700 \mathrm{hPa}, 500 \mathrm{hPa}$, and $300 \mathrm{hPa}$ ); the threshold values were 2 and $1 \mathrm{~K}$, respectively. As for RCM datasets, one of the criteria for identifying tropical cyclones used by Au-Yeung and Chan (2012) was that a temperature anomaly at $300 \mathrm{hPa}$ must be $1 \mathrm{~K}$ greater than the mean temperature within a 15 -degree radius from the vortex center. Huang and Chan (2014) also utilized the same threshold.

Although many schemes for tropical cyclone detection have been reported, a scheme for climate model datasets with a fine grid length (i.e., a grid spacing from $20 \mathrm{~km}$ to $5 \mathrm{~km}$ ) is lacking because these kinds of high-resolution datasets have only recently become available. There are several issues to be solved in order to develop a scheme suitable for the highresolution climate model datasets.

First, thresholds associated with tropical cyclone detection have to be determined for use in highresolution datasets because the threshold values depend on the grid resolution. This problem, however, can be avoided when these thresholds are independent 
of the horizontal resolution. It is therefore desirable to develop a resolution-independent scheme for tropical cyclone detection. Recently, Tory et al. (2013a, b) developed a new method that minimized the resolution dependency of the thresholds. One parameter they used was the Okubo-Weiss-Zeta parameter, a large-scale parameter. This method was designed for coarse-resolution datasets. The performance of the scheme was assessed using relatively coarse-resolution datasets (i.e., $1^{\circ} \times 1^{\circ}$ grid spacing). It was found that realistic spatio-temporal distributions were detectable using the Okubo-Weiss-Zeta parameter. On the other hand, Satake et al. (2013) developed a new method using a technique called neighbor-enclosed area tracking. This technique is based on temporally overlapping areas over a vorticity threshold. In their study, the performance dependence of the proposed scheme on the horizontal resolution of the datasets was examined by comparing the results with $60 \mathrm{~km}$ and $110 \mathrm{~km}$ grid spacings. The results demonstrated that the scheme was relatively independent of the resolution. Although efforts have been undertaken recently, the schemes developed in both of these studies were developed for relatively coarse mesh datasets (i.e., a grid spacing larger than $50 \mathrm{~km}$ ). What has yet to be examined is whether the previously proposed schemes can be applicable to the finer mesh datasets (i.e., a grid spacing from $20 \mathrm{~km}$ to $5 \mathrm{~km}$ ). Schemes for highresolution data sets are desirable.

Second, using the relative vorticity at $850 \mathrm{hPa}$ is difficult because this level is underground in some mountainous regions in Japan, even though this quantity near the vortex center has often been used to detect tropical cyclones in previous schemes. Since the goal of a subsequent study is to investigate future changes in precipitation over Japan associated with tropical cyclones, tropical cyclone tracks over the land in Japan are important.

Third, it is desirable to develop a scheme for cases when the upper-level data are not available due to the limitations of computational and data storage resources. Since the data volumes of RCM and high-resolution GCM datasets are large due to their fine grid spacing and long-term numerical integration, it is possible that only the surface data are provided in order to minimize data storage. Shimura et al. (2017) proposed a tropical cyclone detection method based only on sea level pressure and surface wind speed and found satisfactory performance for their study of extreme wave climates generated by tropical cyclones.

Finally, it is preferable to propose a physically based scheme. The scheme developed in this study is based on the tropical cyclone structure. In particular, the radial gradient and tangential asymmetry were used to extract the tropical cyclones from all the detected vortices. This procedure is similar to a cyclone phase space proposed by Hart (2003). This cyclone phase space was introduced into a scheme for detecting the extratropical transition of tropical cyclones proposed by Zarzycki et al. (2017).

In order to solve these issues, we have proposed a new scheme for detecting tropical cyclones in a gridded dataset produced by high-resolution climate simulations. The goal of this paper is to describe this new scheme and evaluate the detection performance. We show promising results using this scheme. This paper is organized as follows. Section 2 describes the data used in this study. Section 3 presents a new scheme for detecting tropical cyclones in output data provided by RCM simulations. This new scheme consists of two parts: vortex detection and tropical cyclone extraction from the detected vortices. In Section 4, the performance of the tropical cyclone detection scheme is evaluated. Three kinds of RCM datasets were used to assess the performance of the scheme. These datasets include a $20 \mathrm{~km}$ grid dataset forced by reanalysis data, a $20 \mathrm{~km}$ grid dataset forced by an atmospheric general circulation model (AGCM), and a $5 \mathrm{~km}$ grid dataset forced by an AGCM. Section 5 discusses several issues related to the performance of the scheme. These issues include the characteristics of identified tropical cyclones. Case studies using only the surface data and using the gradient over the outer radii were also examined. In addition, the influence of vortex tilt on the tropical cyclone detection scheme was evaluated. Finally, the main results of the study are summarized in Section 6. A companion paper (Watanabe et al. 2018) evaluates the precipitation associated with tropical cyclones in the present climate simulated by a RCM and will analyze the projected precipitation in a warm climate.

\section{Data}

Three kinds of RCM datasets were employed in order to assess the performance of the new tropical cyclone detection scheme from regional climate model data. All datasets were produced by regional climate simulations using the nonhydrostatic regional climate model (NHRCM; Sasaki et al. 2008) developed by the Meteorological Research Institute (MRI) of the Japan Meteorological Agency (JMA), based on the JMA nonhydrostatic model (JMA-NHM; Saito et al. 2006, 2007). The specifications of NHRCM are also described in Nakano et al. (2012) and Murata et al. 
(2015). Data near the lateral boundaries were not used to minimize their impacts.

First, a gridded dataset with $20 \mathrm{~km}$ grid spacing whose boundary conditions were set from reanalysis data (Nosaka et al. 2014) was used so that detected tropical cyclones in the modeled data could be directly compared with observed tropical cyclones. The model domain covered $18^{\circ} \mathrm{N}-47^{\circ} \mathrm{N}$ and $116^{\circ} \mathrm{E}-150^{\circ} \mathrm{E}$ (Fig. 1). The simulation was started on September 1, 2006, and was allowed to run for one year. As for the observed data, the best track data provided by the Regional Specialized Meteorological Center (RSMC)Tokyo were used, except for a part of the extratropical transition. The reanalysis data were produced by the JMA Climate Data Assimilation System (JCDAS) and were the temporally extended version of the Japanese 25-year reanalysis data (JRA-25; Onogi et al. 2007).

Second, a gridded dataset with $20 \mathrm{~km}$ grid spacing that represented the present climate (Kawase et al. 2015; Murata et al. 2016) was used. The model domain covered $18^{\circ} \mathrm{N}-47^{\circ} \mathrm{N}$ and $117^{\circ} \mathrm{E}-153^{\circ} \mathrm{E}$ (Fig. 1). The simulation was started on September 1, 1984, and was allowed to run for 20 years. The boundary conditions for the simulation were provided by a simulation with an AGCM with a $60 \mathrm{~km}$ horizontal resolution (MRI-AGCM3.2H, hereafter referred to as AGCM60; Mizuta et al. 2012).

Third, a gridded dataset with $5 \mathrm{~km}$ grid spacing (Murata et al. 2015) was used. Similar to the second dataset, this dataset also represented the present climate. However, the simulation was conducted using a higher horizontal resolution; the grid spacing was $5 \mathrm{~km}$ instead of $20 \mathrm{~km}$. The four corners of the model domain were $\left(26^{\circ} \mathrm{N}, 113^{\circ} \mathrm{E}\right),\left(15^{\circ} \mathrm{N}, 132^{\circ} \mathrm{E}\right),\left(57^{\circ} \mathrm{N}\right.$, $\left.140^{\circ} \mathrm{E}\right)$, and $\left(38^{\circ} \mathrm{N}, 162^{\circ} \mathrm{E}\right)$ (Fig. 1). The simulation was started on September 1, 1980, and was allowed to run for 20 years. The boundary conditions for the simulation were provided by a simulation with an AGCM with a $20-\mathrm{km}$ horizontal resolution (MRI-AGCM3.2S, hereafter referred to as AGCM20; Mizuta et al. 2012).

\section{Tropical cyclone detection method}

The procedure for detecting tropical cyclones in the RCM results is divided into two parts: vortex detection and the extraction of tropical cyclones from the detected vortices. The two procedures are described below.

\subsection{Vortex detection}

First, vortices including tropical cyclones are identified in the RCM results. A vortex is assumed to satisfy all of the following criteria:

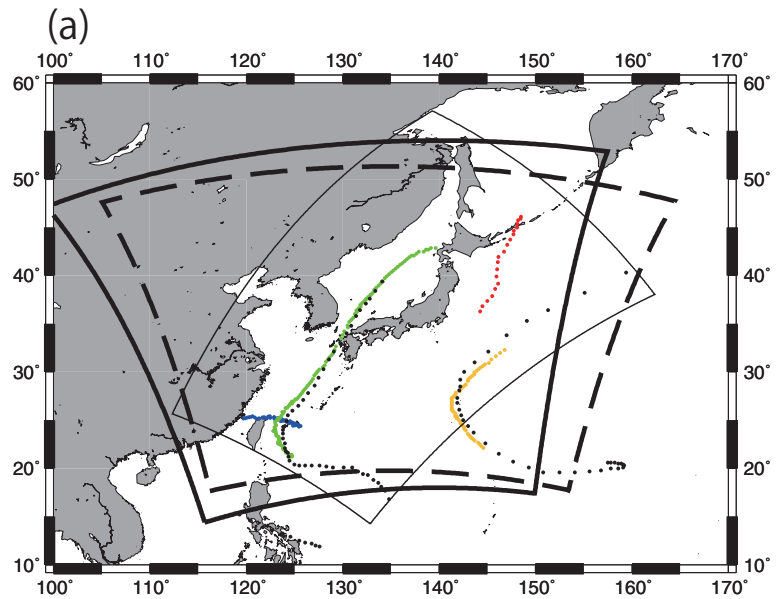

(b)

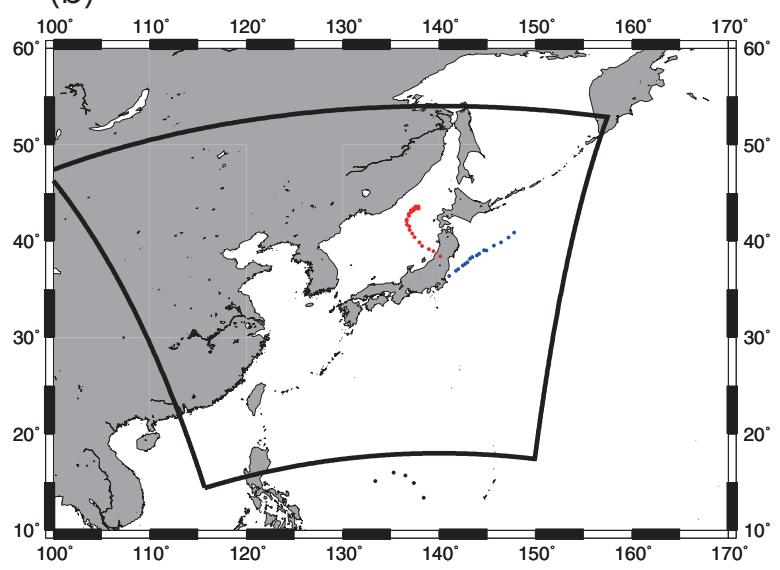

Fig. 1. Examples of detected vortices in (a) fall (September 1, 2006 to November 30, 2006) and (b) winter (December 1, 2006 to February 28, 2007). The black dots represent the observed tropical cyclones (six-hour interval) and the colored dots represent the simulated vortices (one-hour interval). The green and yellow dots are for the simulated typhoons Shanshan (2006) and Yagi (2006), respectively. The NHRCM20 domain (reanalysis boundary) is shown in (a) and (b) by the thick solid lines. The NHRCM20 (AGCM60 boundary) and the NHRCM05 (AGCM20 boundary) domains are also shown in (a) by the thick dashed lines and the thin solid lines, respectively.

1) The local minimum sea level pressure (MSLP) is lower than a certain threshold. In this study, the threshold value was set to $1000 \mathrm{hPa}$. This local minimum is defined as the vortex center.

2) The maximum surface wind speed, within a square area whose center corresponds to the vortex center, 
is greater than $17 \mathrm{~m} \mathrm{~s}^{-1}$. A square $200 \mathrm{~km}$ on a side was set in this study. The maximum surface wind speed can be realistically represented in NHRCM because of its higher resolution.

3) There are no other vortices within the area defined above. When vortices exist in the area, the vortex that has the lowest MSLP is selected. This criterion allows the scheme to detect the vortex without the need for a spatial filter.

4) The method of vortex tracking was as follows: after a certain period, when a vortex existed within a square area whose center corresponded to the original vortex center before the period, these two vortices were assumed to be identical. A period of one hour and a square $120 \mathrm{~km}$ on a side were set in this study. Setting the period of one hour is intended to examine one-hour accumulated precipitation.

5) The consecutive period during which the three criteria above (i.e., the items 1, 2, 3, and 4) are satisfied is longer than or equal to a certain threshold. The threshold was set at 12 hours in this study.

\subsection{Tropical cyclone extraction from the detected vortices}

Next, the tropical cyclones were extracted from the detected vortices in the RCM results. The criterion for the extraction was based on the layer thickness between the middle and upper troposphere; $500 \mathrm{hPa}$ and $300 \mathrm{hPa}$ were set in this study (hereafter referred to as $D$ ). In terms of $D$, quantities related to the radial gradient $(G)$ and the tangential asymmetry $(A)$ of $D$ are defined as follows:

$$
\begin{aligned}
G & =\frac{1}{4} \sum_{i=1}^{4}\left(\frac{D_{i}-D_{0}}{R}\right), \\
A & =\frac{\left|D_{1}-D_{2}\right|+\left|D_{2}-D_{3}\right|+\left|D_{3}-D_{4}\right|+\left|D_{4}-D_{1}\right|}{2 \pi R} .
\end{aligned}
$$

$G$ represents the radial gradient of $D$ between the vortex center and at a radius $(R)$, and $A$ represents the tangential asymmetry of $D$ along the circumference of $R$. In order to reduce the computational cost, values of $D$ were included only at five grid points in Eqs. (1) and (2): at the vortex center $\left(D_{0}\right)$, at $0\left(D_{1}\right), 90\left(D_{2}\right)$, $180\left(D_{3}\right)$, and $270\left(D_{4}\right)$ degrees in a clockwise direction with respect to the $\mathrm{Y}$ axis. In this study, $R$ was set at $300 \mathrm{~km}$. The indices, $G$ and $A$, are temporally averaged over the period of the vortex lifetime. The relationship between $G$ and $A$ were utilized to extract the tropical cyclones from all the detected vortices. This procedure is graphically shown in Section 4. It should be noted that a scheme for the extratropical transition of a tropical cyclone will be added in a companion paper (Watanabe et al. 2018).

\section{Evaluation of the tropical cyclone detection scheme}

In order to assess the performance of the new tropical cyclone detection scheme from regional climate model data, three kinds of RCM datasets, as mentioned in Section 2, were utilized. The results of the performance assessment using each dataset are described below.

\subsection{The $20 \mathrm{~km}$ grid dataset forced by reanalysis data}

First, the vortex detection performance was examined. Examples for fall and winter are displayed in Fig. 1. In fall (Fig. 1a), there were two tropical cyclones around Japan: Typhoon Shanshan (2006) and typhoon Yagi (2006). Generally, Shanshan and Yagi were located west and east of Japan, respectively.

Both the Shanshan and Yagi tracks were captured in the $20 \mathrm{~km}$ grid model data (Fig. 1a). For Shanshan, its northeastward movement is represented in the simulation although the detected vortex appeared later and disappeared earlier than in the observations. Similarly, the detected track of Yagi corresponded to observations except for the southern and northern parts; in these parts, an outer region of a tropical cyclone (i.e., $R$ is greater than $300 \mathrm{~km}$ ) is outside the model domain. Since all vortices were detected, vortices other than tropical cyclones were also included in Fig. 1a. Vortices other than tropical cyclones were also detected in winter. In Fig. 1b, two vortices were detected around Japan, whereas there were no observed tropical cyclones. This result indicates a requirement for the extraction of tropical cyclones from all simulated vortices.

Next, tropical cyclones were distinguished from other vortices by the relationship between the two indices mentioned above: $G$ and $A$. The scatterplot of $A$ versus $G$ is shown in Fig. 2. It should be noted that these two indices are temporally averaged over the period of the vortex lifetime. A simulated vortex was judged to be a tropical cyclone when the distance between the vortex and an observed tropical cyclone, averaged over the lifetime of the vortex, was within $500 \mathrm{~km}$. The scatterplot revealed that data for the tropical cyclones were located around the lower left of Fig. 2, compared with vortices other than tropical cyclones. That is, the magnitude of $G$ for tropical cyclones is greater than for the other vortices $(G$ is generally negative); $A$ for tropical cyclones is smaller than for the other vortices. This result reflects the 


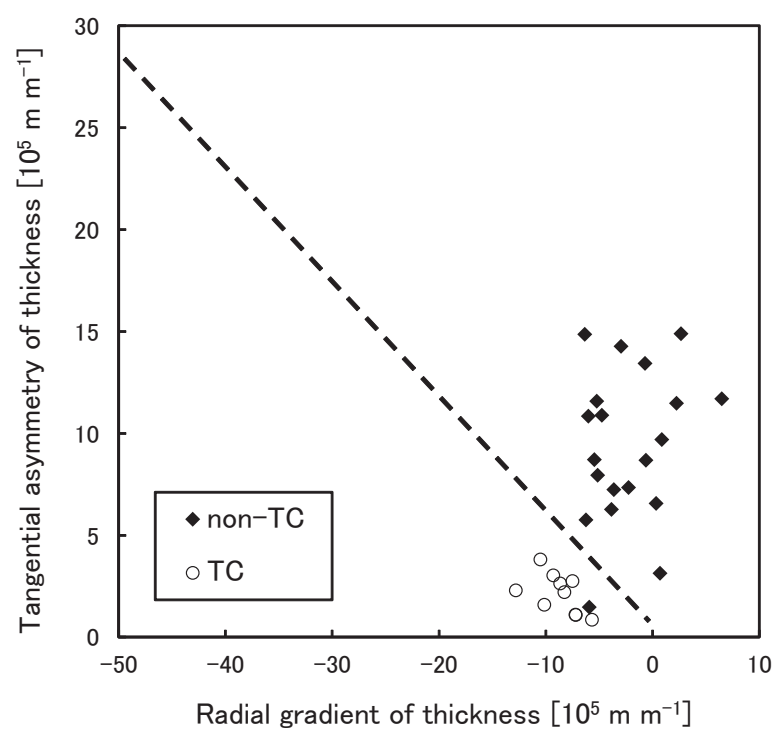

Fig. 2. The radial gradient, between the center and the $300 \mathrm{~km}$ radius, of the thickness between 500 $\mathrm{hPa}$ and $300 \mathrm{hPa}$ versus the tangential asymmetry of the thickness at the $300 \mathrm{~km}$ radius. The open circles and filled diamonds denote tropical cyclones and others (mainly extratropical cyclones), respectively. The $20 \mathrm{~km}$ grid dataset forced by the reanalysis data was used.

warm core structure of tropical cyclones. A warm core, typically located in the middle to upper troposphere, has a structure of negative $G$ because the layer between thicknesses of $500 \mathrm{hPa}$ and $300 \mathrm{hPa}$ is thicker near the vortex center than the outer radii. The other index, $A$, has an approximate value of zero because of the axisymmetric structure of tropical cyclones. In contrast, vortices other than tropical cyclones, mainly extratropical cyclones, have neither a warm core structure nor an axisymmetric structure. A line that differentiates the two categories is shown in Fig. 2. The slantwise line was intended to divide subtropical lows (e.g., Ogura et al. 2005, 2009), located in a region of small magnitudes of $G$ and $A$, into the tropical and extratropical cyclones on the basis of their structure. The equation of the line is as follows:

$$
\frac{A}{G}=-\frac{14}{25}=-0.56
$$

The lefthand side of Eq. (3) represents an asymmetry index normalized by $G$. The magnitude of $A$ is about half the magnitude of $G$ in this case, although the specific value (i.e., -0.56 ) is rather arbitrary. This value may depend on climate models.

The results demonstrate that the simulated tropical cyclones were proficiently detected, considering that there were none missed and only one false alarm. This falsely alarmed system was located in a subtropical area and did not have a surface front, thereby leading to the judgment that the system was in the tropical cyclone category.

\subsection{The $20 \mathrm{~km}$ grid dataset forced by the AGCM60}

Tropical cyclones were detectable in the $20-\mathrm{km}$ grid RCM dataset, whose boundary conditions were AGCM60 results, by using the criteria mentioned above. The relationship between $G$ and $A$ seemed to hold true for this dataset (Fig. 3a). The plotted points could be classified into two categories. In the first category, the magnitude of $G$ (generally negative) was relatively large, and $A$ was near zero. In contrast, in the second category, the magnitude of $G$ was near zero and $A$ was relatively large. The first and second category corresponded to tropical cyclones and extratropical cyclones, respectively. Result verifications were not performed due to the limits of the present climate simulation data, in which a simulated tropical cyclone does not correspond one to one with the observed cyclone. The range of $G$ was wider than in the reanalysis forced RCM data (Fig. 2). The maximum magnitude of $G$ almost reached $2.5 \times 10^{6} \mathrm{~m} \mathrm{~m}^{-1}$ in Fig. $3 \mathrm{a}$, whereas it was below $1.5 \times 10^{6} \mathrm{~m} \mathrm{~m}^{-1}$ in Fig. 2 . This is probably due to a difference in period data cover that was 20 years in Fig. 3a and 1 year in Fig. 2. An intensified tropical cyclone that has a large magnitude of $G$ can be simulated in a long-term climate experiment, such as in Fig. $3 \mathrm{a}$.

\subsection{The $5 \mathrm{~km}$ grid dataset forced by the AGCM20}

Similar to the $20 \mathrm{~km}$ grid datasets, tropical cyclones were detectable in the $5 \mathrm{~km}$ grid RCM dataset, whose boundary conditions were the AGCM20 results, by using the criteria described above. The relationship between $G$ and $A$ seemed to also hold true for this dataset (Fig. 3b). Similarly, there were two categories identified as shown in Fig. 3b. The data in one category had greater magnitudes of $G$ and near-zero values for $A$, whereas those in the other category had near-zero $G$ and greater $A$ values. The former and latter categories corresponded to tropical cyclones and extratropical cyclones, respectively.

The $A$ index was less dependent on the horizontal resolution compared to the $G$ index. The range of $A$ in the $5 \mathrm{~km}$ grid dataset (Fig. 3b) was similar to the 20 $\mathrm{km}$ grid dataset (Fig. 3a). The maxima of $A$ for both 
(a)

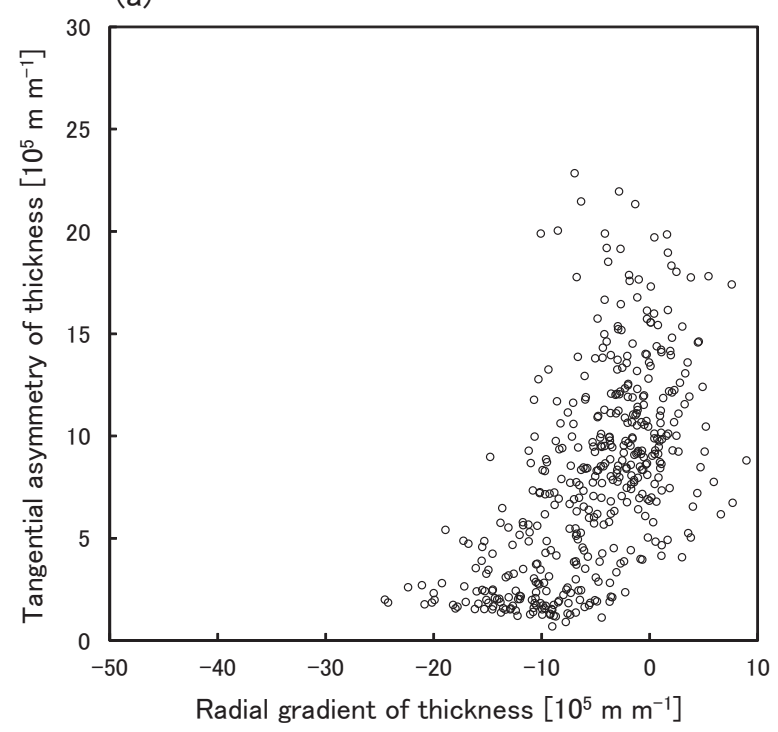

(b)

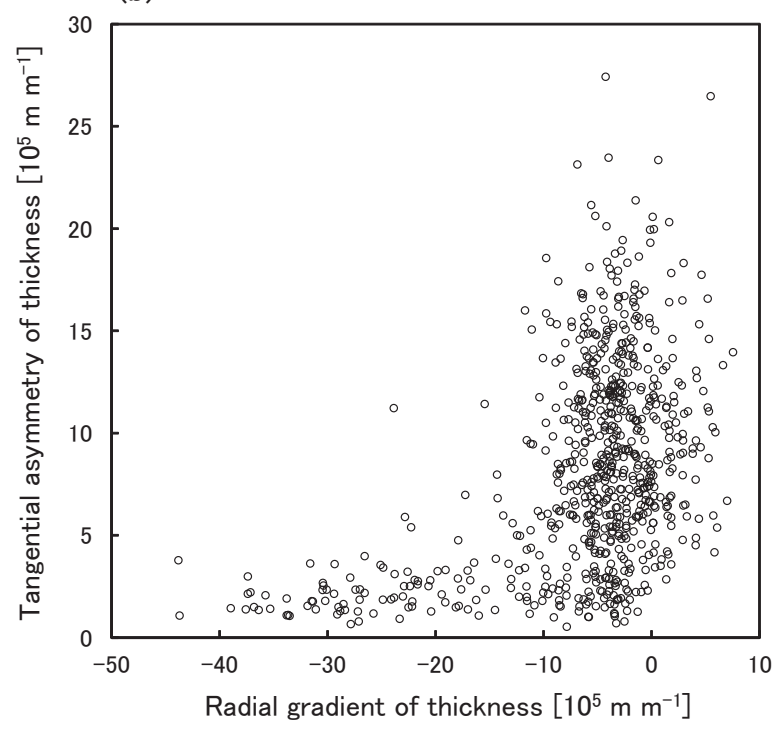

Fig. 3. The radial gradient, between the center and the $300 \mathrm{~km}$ radius, of the thickness between 500 $\mathrm{hPa}$ and $300 \mathrm{hPa}$ versus the tangential asymmetry of the thickness at the $300 \mathrm{~km}$ radius. (a) The 20 $\mathrm{km}$ grid dataset forced by the AGCM60 and (b) the $5 \mathrm{~km}$ grid dataset forced by the AGCM20.

were below $3.0 \times 10^{6} \mathrm{~m} \mathrm{~m}^{-1}$ (Figs. 3a, b). In contrast, the range of $G$ in the former dataset was wider than in the latter. The maximum of the magnitude of $G$ exceeds $4.0 \times 10^{6} \mathrm{~m} \mathrm{~m}^{-1}$ in Fig. $3 \mathrm{~b}$, whereas it was below $2.5 \times 10^{6} \mathrm{~m} \mathrm{~m}^{-1}$ in Fig. $3 \mathrm{a}$.

In terms of tropical cyclones, the weak dependence of $A$ on the horizontal resolution is attributed to an adequate representation of the outer radius of a tropical cyclone in both the $20 \mathrm{~km}$ and $5 \mathrm{~km}$ grid datasets. For proper representation near the vortex center, on the other hand, the $20 \mathrm{~km}$ grid spacing was insufficient for a tropical cyclone. This is because there is a steep temperature gradient, and hence thickness, near the vortex center. To resolve this fine structure, a finer grid spacing (e.g., $5 \mathrm{~km}$ ) is required. For this reason, the range of $G$ in the $5 \mathrm{~km}$ grid datasets was different from the $20 \mathrm{~km}$ grid dataset.

From a practical point of view, a threshold to distinguish between tropical and extratropical cyclones needs to be determined. Specifically, the most favorable line that differentiates the two categories in Figs. 3a, b, similar to that in Fig. 2, should be determined so that the number of identified tropical cyclones becomes nearly equal to the observed climate data. This issue will be discussed in detail in a companion paper (Watanabe et al. 2018).

\section{Discussion}

\subsection{Comparison with Hart (2003)}

The relationship between the radial gradient and the outer asymmetry of mid- to upper- tropospheric thicknesses for tropical cyclones has been clarified. The former and latter quantities are represented by the $G$ and $A$ indices, respectively. In Fig. 3b, the magnitude range of $G$ is wider than $A$ for the identified tropical cyclones. This means that the degree of the thickness asymmetry at the outer radius is less dependent on the intensity of a tropical cyclones compared with the radial gradient of the thickness. It should be noted that an intensified tropical cyclone tends to have a large magnitude of $G$.

Scatter diagrams of the relationship between $G$ and $A$ (Figs. 3a, b) are similar to a cyclone phase space proposed by Hart (2003). This cyclone phase space was designed to identify the tropical cyclone phases, namely for tropical, extratropical, and hybrid cyclones. The parameters used in this cyclone phase space are the vertical derivative of the horizontal height gradient and the thickness asymmetry. The former parameter that corresponds to $G$ in the present study distinguishes the warm core structure from the cold core structure, although the thermal wind relationship is used instead of the direct calculation of the radial gradient. On the other hand, the latter parameter, equivalent to $A$ in the present study, discriminates between the frontal and nonfrontal phase. The cyclone phase space, therefore, is different from the scatter diagram in the present study, although the underlying theories are the 
same. In addition, the cyclone phase space classifies a vortex into categories at a specific time point, whereas $G$ and $A$ in the present study are temporally averaged over the vortex lifetime.

\subsection{A case using only the surface data}

Using only the surface data, tropical cyclones can be detected even when the upper data are unavailable, although the accuracy is slightly decreased. In these cases, sea level pressure is used instead of the thickness between $500 \mathrm{hPa}$ and $300 \mathrm{hPa}$ in the scatter diagrams for the relationship between $G$ and $A$. Figure $4 \mathrm{a}$ is a similar diagram to Fig. 2 but uses sea level pressure data. Similar to Fig. 2, there are two groups of data, representing tropical and extratropical cyclones. The data for one group, however, mixes with the other group. The mixture of the two categorized data is also found in the $5 \mathrm{~km}$ grid dataset, although the range of $G$ is relatively wider (Fig. 4b). These results can decrease the skill of the model for detecting tropical cyclones. Nevertheless, this detection method has the possibility of detecting tropical cyclones, even when only the surface data is available, which is not necessarily uncommon for a huge dataset related to high-resolution climate simulations.

The relatively low skill in detecting tropical cyclones using only the surface data is attributable to the structure of tropical and extratropical cyclones at the surface. Intensified extratropical cyclones can have a large magnitude of $G$ and a small $A$, similar to those for tropical cyclones, leading to a mixture of the data for tropical and extratropical cyclones in some graph regions. In contrast, the data mixing appears less frequently when using the upper-level data because of the warm core structure that leads to a large magnitude of $G$ and a small $A$ that does not usually occur in extratropical cyclones.

\subsection{A case using the gradient over the outer radii}

The resolution dependence of the detection method can be improved by substituting radial gradients over the outer radii for those data points near the vortex center. The range of the magnitude of $G$ varies with the horizontal resolution, as described above (Figs. $3 a, b)$. This dependence on resolution can be reduced when the thickness at the outer radii is used. For example, Figs. 5a, b are the same type of diagrams as Figs. 3a, b, respectively, but use the radial gradient between $300 \mathrm{~km}$ and $400 \mathrm{~km}$ instead of between $0 \mathrm{~km}$ and $300 \mathrm{~km}$. In Fig. 5, the maximum magnitude of $G$ is about $2.0 \times 10^{6} \mathrm{~m} \mathrm{~m}^{-1}$ for both the $20 \mathrm{~km}$ and 5 $\mathrm{km}$ grid datasets, indicating that $G$ was nearly inde-

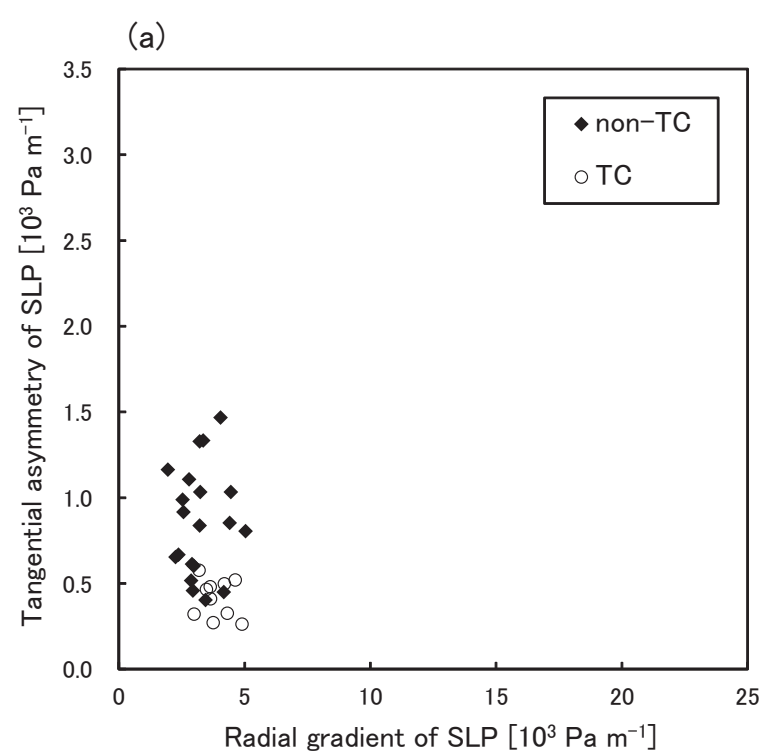

(b)

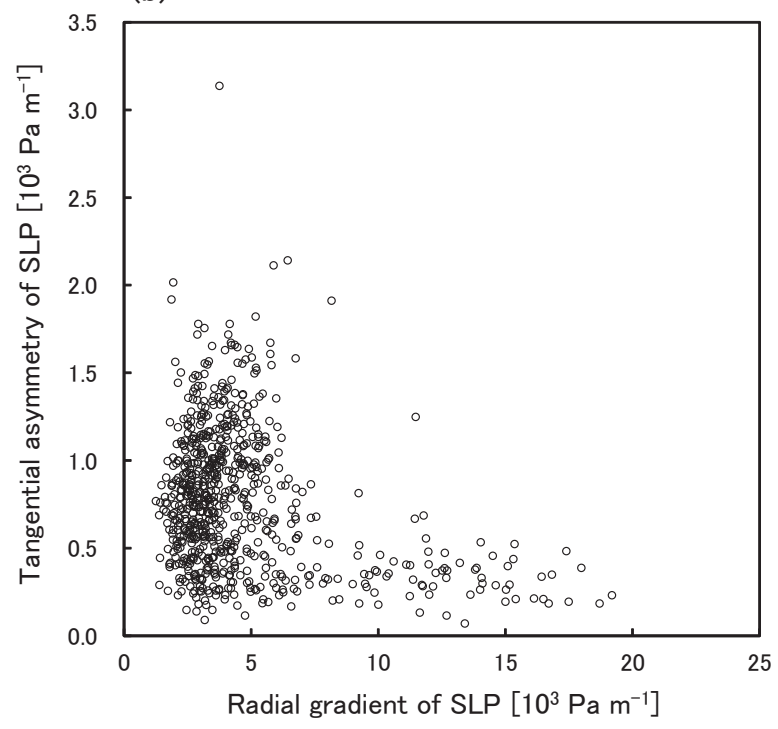

Fig. 4. (a) Same as Fig. 2, but sea level pressure was used instead of the thickness between 500 hPa and 300 hPa. (b) Same as Fig. 3b, but sea level pressure was used instead of the thickness between $500 \mathrm{hPa}$ and $300 \mathrm{hPa}$.

pendent of the horizontal resolution. It is possible, however, that the distinction between tropical and extratropical cyclones is insufficient, particularly in the graph region where both $G$ and $A$ are nearly zero. In this graph region, the mixture of data for tropical and extratropical cyclones can occur. This is because the differences in $G$ over the outer radii between 


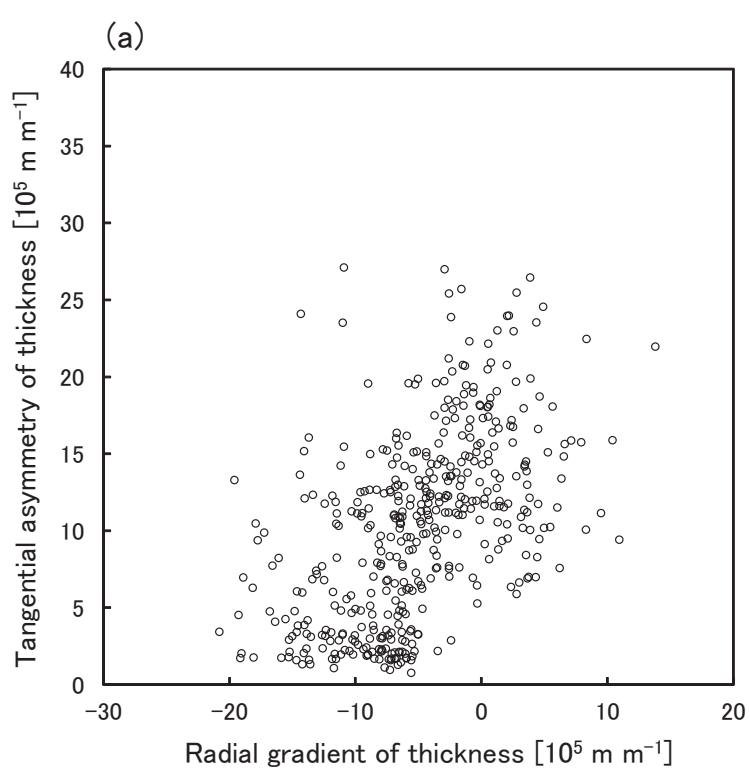

(b)

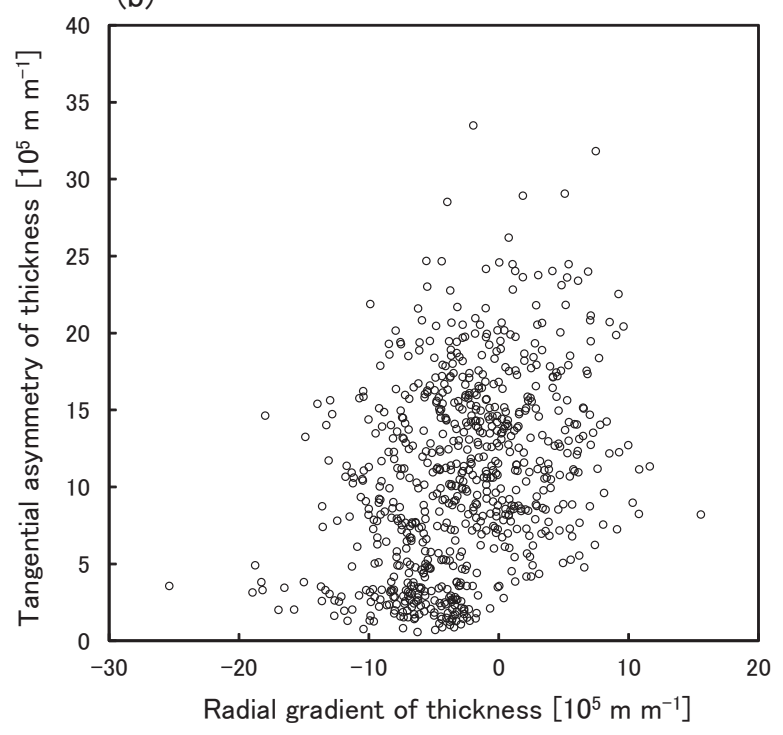

Fig. 5. Same as Fig. 3, but the horizontal axis represents the radial gradient between the $300 \mathrm{~km}$ and $400 \mathrm{~km}$ radii, instead of between the center and the $300 \mathrm{~km}$ radius.

tropical and extratropical cyclones is smaller than near the vortex center. Further study is needed to utilize the radial gradient of the thickness over the outer radii of a tropical cyclone.

\subsection{Influence of vortex tilt}

An issue associated with vortex tilt is addressed

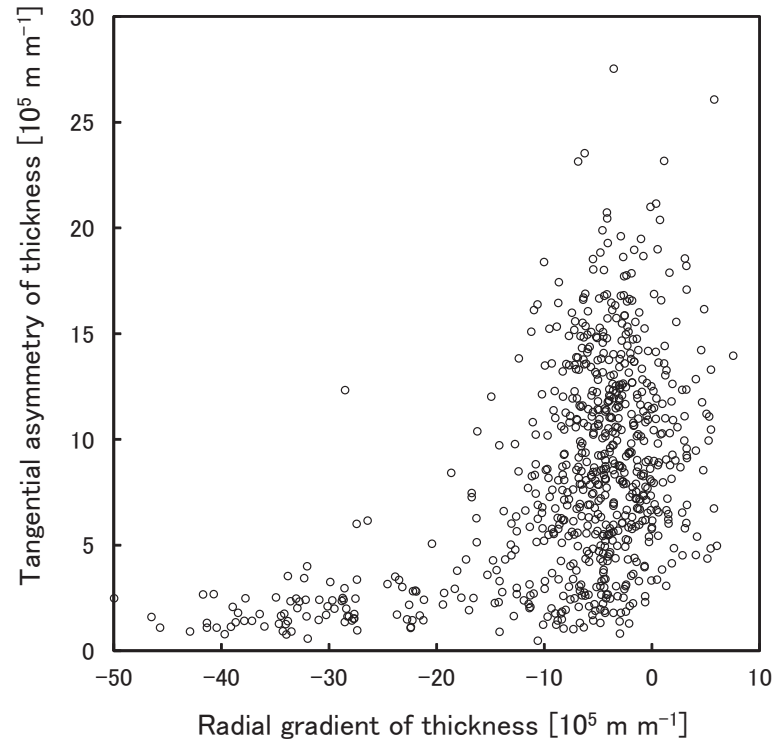

Fig. 6. Same as Fig. 3b, but including the effects of vortex tilt.

here. Several tropical cyclones show that the axes of their vortex center tilt with height due to vertical wind shear. In order to seek the simplest possible detection method, this effect was not considered for the detection of tropical cyclones described above. That is, the vortex center at the upper level is assumed to coincide with the surface for simplicity. In order to examine the effects of vortex tilt on the performance of the tropical cyclone detection, a sensitivity test including this effect was conducted. In this test, the vortex center at an upper level was independently determined. Specifically, the vortex center at $500 \mathrm{hPa}$ was defined as the local minimum of the geopotential height, where the horizontal distance between this minimum and the original were nearest within a square area. A square $100 \mathrm{~km}$ on a side area was set in this study. Using the vortex center at $500 \mathrm{hPa}$, the thickness between 500 $\mathrm{hPa}$ and $300 \mathrm{hPa}$ was calculated. When there was no local minimum of the geopotential height at $500 \mathrm{hPa}$, a no-tilt assumption was made.

The tropical cyclone detection methods in this study were essentially unaffected by the vortex tilt of the tropical cyclones. This contention is supported by the sensitivity test mentioned above. Figure 6 is the same type of diagram as Fig. $3 \mathrm{~b}$ but includes the vortex tilt effect. Comparing Fig. 6 to Fig. $3 \mathrm{~b}$ reveals that the data distributions in both figures are nearly identical, indicating that the former is able to capture the essential structure of tropical cyclones. One possibility 
(a)

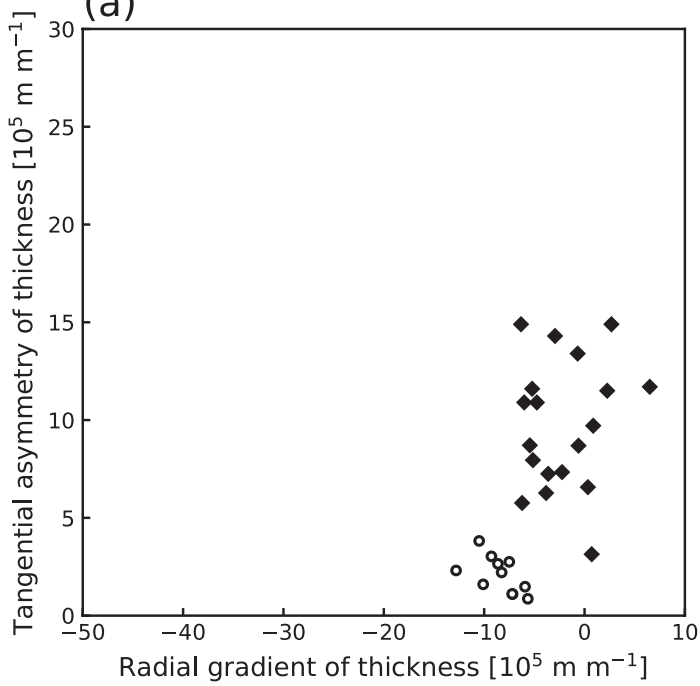

(b)
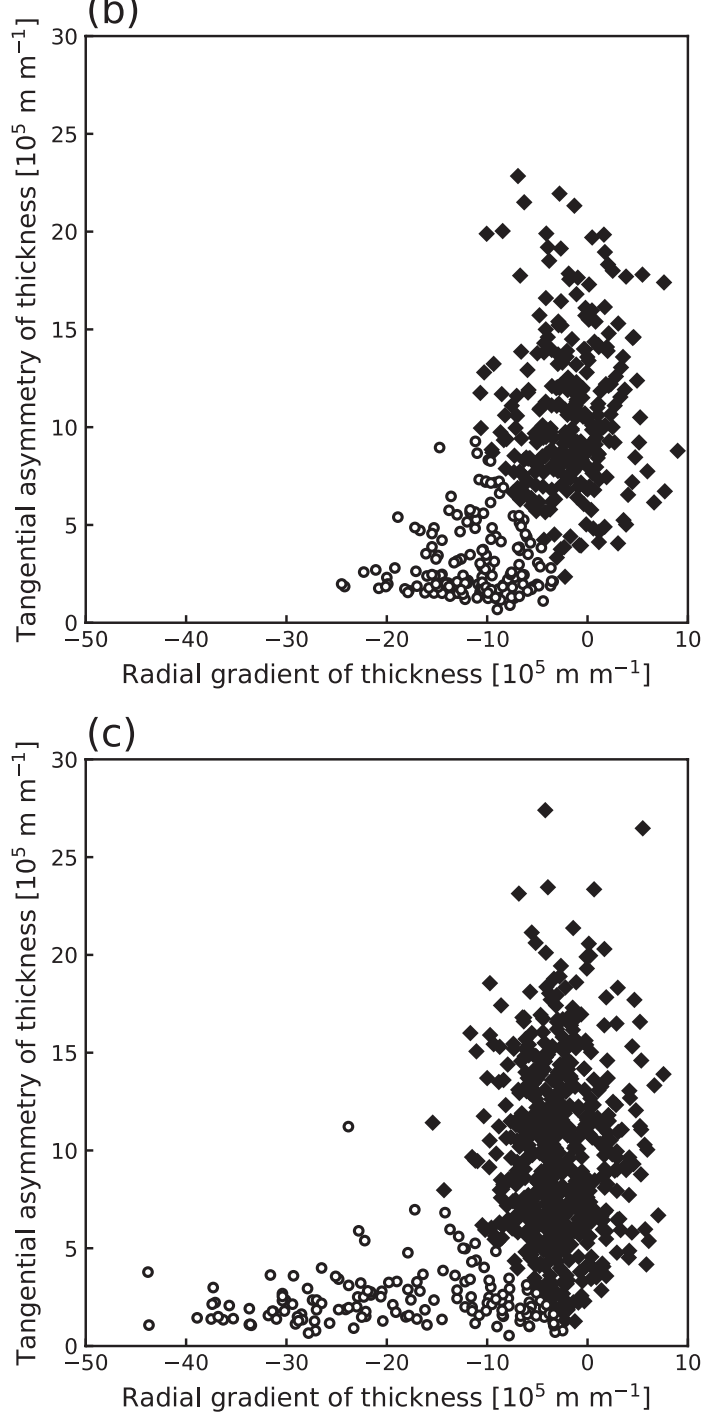

for this result is that $G$ and $A$ are temporally averaged over the vortex lifetime, and this can reduce the effects of vortex tilt.

\subsection{Cluster analysis}

A cluster analysis was performed on the three datasets mentioned above in order to find indirect evidence that the presented tropical cyclone detection scheme is reasonable. The cluster analysis used in this study is an agglomerative method, a kind of hierarchical clustering (Wilks 2011). The distance measurement used here is called the cosine distance. A distance between two clusters is defined as the mean distance between all pairs of data in the two clusters (called the "average linkage").

The cluster analysis suggested that the scheme proposed in this study is skillful enough to detect tropical cyclones. Figures $7 \mathrm{a}-\mathrm{c}$ are the same type of diagrams as Figs. 2, 3a, and 3b, respectively, but use the cluster analysis instead of the presented detection method. Comparing Fig. 7 a to Fig. 2 reveals that a group of data in Fig. 7a (open circles) corresponds to the category of tropical cyclones found in Fig. 2 (open circles), and the other group in Fig. 7a (filled diamonds) corresponds to the extratropical cyclones found in Fig. 2 (filled diamonds). More specifically, the threshold line in Fig. 2 can also be applied in Fig. $7 \mathrm{a}$. This nearly equal classification result provides indirect evidence suggesting the rationality of the presented detection scheme. In addition, comparing Figs. $7 \mathrm{~b}, \mathrm{c}$ to Fig. 7a reveals that the classification results in Figs. 7b, c are similar to Fig. 7a. This result suggests that the threshold for dividing the two categories does not vary significantly with the horizontal resolution.

\section{Summary and concluding remarks}

A new scheme for detecting tropical cyclones in a gridded dataset was developed. The aim was to use high-resolution climate model datasets, namely with grid spacings of $20 \mathrm{~km}$ and $5 \mathrm{~km}$, with a variety of horizontal resolutions and with no change in the

Fig. 7. (a) Same as Fig. 2, but a cluster analysis was used for classifying data into two groups, denoted by the open circles and filled diamonds. The $20 \mathrm{~km}$ grid dataset forced by the reanalysis data was used. (b) Same as Fig. 7a, but the $20 \mathrm{~km}$ grid dataset forced by the AGCM60 was used. (c) Same as Fig. 7a, but the $5 \mathrm{~km}$ grid dataset forced by the AGCM 20 was used. 
thresholds of the scheme. This scheme contains several characteristic features. One of them is that the scheme does not utilize the relative vorticity at 850 $\mathrm{hPa}$ that has often been used in other schemes because determining a threshold for identifying tropical cyclones is dependent on the horizontal resolution and importantly, $850 \mathrm{hPa}$ is underground in some mountainous regions, such as central Japan. The latter reason is also important considering that our main target in a companion paper (Watanabe et al. 2018) is the precipitation over mountainous areas of Japan associated with tropical cyclones. Another feature of this scheme is the possibility of detecting tropical cyclones using only the surface data when upper-level data are not available. Note that extratropical cyclones with small asymmetry can be wrongly identified as a tropical cyclone using only the surface data. Moreover, the classification method used for extracting vortices related to tropical cyclones and extratropical cyclones is based on a physically plausible technique, similar to the phase space proposed by Hart (2003). In our scatterplots, the radial gradient and the tangential asymmetry of the layer thickness between levels at the middle and upper troposphere were used.

The performance of the tropical cyclone detection scheme was evaluated using three kinds of RCM datasets, a $20 \mathrm{~km}$ grid dataset forced by reanalysis data, a $20 \mathrm{~km}$ grid dataset forced by an AGCM, and a $5 \mathrm{~km}$ grid dataset forced by an AGCM. Using the first dataset allowed us to compare the detected tropical cyclones with actual observations. The second dataset had the same horizontal resolution as the first but represented the present climate. In this case, comparisons between the simulated and observed tropical cyclones should be made statistically because comparing a detected tropical cyclone with an observed cyclone individually is meaningless. The third dataset also used the present climate dataset but at a higher resolution.

Evaluation of the three datasets demonstrated that the scheme proposed in this study can skillfully detect tropical cyclones. In the first dataset, a one-by-one comparison between the simulated and observed tropical cyclones showed that none were missed and there was only one false alarm. The simulated data were classified into two categories: tropical cyclone and extratropical cyclones. The former data group have a greater radial gradient and less tangential asymmetry in terms of layer thickness between $500 \mathrm{hPa}$ and 300 $\mathrm{hPa}$. In contrast, in the latter data group, the gradient is relatively low, and the degree of asymmetry is higher. Simulated tropical cyclones were also detectable in the second and third datasets. For both datasets, the data could be classified into the two groups similar to those for the first dataset, indicating that the scheme has the ability to categorize simulated vortices into tropical and extratropical cyclones.

The categorized data reflects the characteristics of the detected tropical and extratropical cyclones. The relationship between the radial gradient and the tangential asymmetry of the mid- to upper- tropospheric thickness for tropical cyclones has been clarified. It was found that the degree of the tangential asymmetry of the thickness at the outer radius is less dependent on the intensity of a tropical cyclone compared with the radial gradient of the thickness.

The skillful detection of tropical cyclones using the developed scheme is supported indirectly by a cluster analysis. The result obtained from the cluster analysis was nearly equal to results obtained from the method used in the presented scheme. That is, the simulated data were categorized into two groups: tropical and extratropical cyclones. It was also found that the threshold for dividing the two groups does not vary significantly with the horizontal resolution.

The detection scheme developed in this study is only weakly dependent on the horizontal resolution of a dataset. This weak dependence is attributed to one parameter in the two-dimensional scatter diagram. This parameter is the tangential asymmetry of the layer thickness between $500 \mathrm{hPa}$ and $300 \mathrm{hPa}$ at the outer radii that is not strongly influenced by horizontal resolution. As for the other parameter, the radial gradient of the thickness, it was found that using the quantity over the outer radii instead of near the vortex center has the potential for developing a resolutionindependent detection method.

Several issues relevant to the performance of the tropical cyclone detection scheme were discussed. One of them is associated with the vortex tilt of tropical cyclones. A sensitivity test revealed that the detection of tropical cyclones in this study was essentially unaffected by the vortex tilt. Another issue is associated with data availability. Even when only surface data is available, as is often the case with high-resolution climate datasets, the scheme can reliably detect tropical cyclones, although the detection skill does not exceed that when the upper data are used.

Our main research target was to analyze the projected precipitation over Japan associated with tropical cyclones using high-resolution simulation results, as mentioned above. A companion paper (Watanabe et al. 2018) evaluates the precipitation accompanying tropical cyclones in the present climate and analyzes the projected precipitation in a warm climate. 


\section{Acknowledgments}

This research was supported by JSPS KAKENHI under Grant Number JP16K00526. A portion of the data was supplied by the SOUSEI and TOUGOU programs of the Ministry of Education, Culture, Sports, Science, and Technology (MEXT) of Japan. Another portion of the datasets used for this study was provided from the Japanese 25-year Reanalysis (JRA-25), the cooperative research project carried out by the Japan Meteorological Agency (JMA), and the Central Research Institute of Electric Power Industry (CRIEPI).

\section{References}

Au-Yeung, A. Y. M., and J. C. L. Chan, 2012: Potential use of a regional climate model in seasonal tropical cyclone activity predictions in the western North Pacific. Climate Dyn., 39, 783-794.

Bengtsson, L., H. Böttger, and M. Kanamitsu, 1982: Simulation of hurricane-type vortices in a general circulation model. Tellus, 34, 440-457.

Hart, R. E., 2003: A cyclone phase space derived from thermal wind and thermal asymmetry. Mon. Wea. Rev., 131, 585-616.

Huang, W.-R., and J. C. L. Chan, 2014: Dynamical downscaling forecasts of western North Pacific tropical cyclone genesis and landfall. Climate Dyn., 42, 22272237.

IPCC, 2013: Climate Change 2013: The Physical Science Basis. Contribution of Working Group I to the Fifth Assessment Report of the Intergovernmental Panel on Climate Change. Stocker, T. F., D. Qin, G.-K. Plattner, M. M. B. Tignor, S. K. Allen, J. Boschung, A. Nauels, Y. Xia, V. Bex, and P. M. Midgley (eds.), Cambridge University Press, 1535 pp.

Kanada, S., K. Tsuboki, H. Aiki, S. Tsujino, and I. Takayabu, 2017: Future enhancement of heavy rainfall events associated with a typhoon in the midlatitude regions. SOLA, 13, 246-251.

Kawase, H., H. Sasaki, A. Murata, M. Nosaka, and N. N. Ishizaki, 2015: Future changes in winter precipitation around Japan projected by ensemble experiments using NHRCM. J. Meteor. Soc. Japan, 93, 571-580.

Miyamoto, Y., Y. Kajikawa, R. Yoshida, T. Yamaura, H. Yashiro, and H. Tomita, 2013: Deep moist atmospheric convection in a subkilometer global simulation. Geophys. Res. Lett., 40, 4922-4926.

Mizuta, R., H. Yoshimura, H. Murakami, M. Matsueda, H. Endo, T. Ose, K. Kamiguchi, M. Hosaka, M. Sugi, S. Yukimoto, S. Kusunoki, and A. Kitoh, 2012: Climate simulations using MRI-AGCM3.2 with 20-km grid. $J$. Meteor. Soc. Japan, 90A, 233-258.

Murakami, H., and M. Sugi, 2010: Effect of model resolu- tion on tropical cyclone climate projections. SOLA, $\mathbf{6}$, $73-76$.

Murata, A., H. Sasaki, H. Kawase, M. Nosaka, M. Oh'izumi, T. Kato, T. Aoyagi, F. Shido, K. Hibino, S. Kanada, A. Suzuki-Parker, and T. Nagatomo, 2015: Projection of future climate change over Japan in ensemble simulations with a high-resolution regional climate model. SOLA, 11, 90-94.

Murata, A., H. Sasaki, H. Kawase, and M. Nosaka, 2016: Identification of key factors in future changes in precipitation extremes over Japan using ensemble simulations. Hydrol. Res. Lett., 10, 126-131.

Nakano, M., S. Kanada, and T. Kato, 2010: Statistical analysis of simulated direct and indirect precipitation associated with typhoons around Japan using a cloudsystem resolving model. Hydrol. Res. Lett., 4, 6-10.

Nakano, M., T. Kato, S. Hayashi, S. Kanada, Y. Yamada, and K. Kurihara, 2012: Development of a 5-km-mesh cloud-system-resolving regional climate model at the Meteorological Research Institute. J. Meteor. Soc. Japan, 90A, 339-350.

Nosaka, M., H. Sasaki, A. Murata, and H. Kawase, 2014: Comparison of bias correction methods in terms of winds simulated by a regional climate model. Proceeding of the 2014 Autum Meeting of Meteorological Society of Japan, 359-359 (in Japanese).

Ogura, Y., H. Niino, R. Kumabe, and S. Nisimura, 2005: Evolution of a typhoon-like subtropical low causing severe weather over the Kanto area on 13 October 2003. J. Meteor. Soc. Japan, 83, 531-550.

Ogura, Y., R. Kumabe, and S. Nisimura, 2009: Initiation and evolution of a subtropical low observed near the Japan islands. J. Meteor. Soc. Japan, 87, 941-957.

Onogi, K., J. Tsutsui, H. Koide, M. Sakamoto, S. Kobayashi, H. Hatsushika, T. Matsumoto, N. Yamazaki, H. Kamahori, K. Takahashi, S. Kadokura, K. Wada, K. Kato, R. Oyama, T. Ose, N. Mannoji, and R. Taira, 2007: The JRA-25 Reanalysis. J. Meteor. Soc. Japan, 85, 369432.

Oouchi, K., J. Yoshimura, H. Yoshimura, R. Mizuta, S. Kusunoki, and A. Noda, 2006: Tropical cyclone climatology in a global-warming climate as simulated in a 20 km-mesh global atmospheric model: Frequency and wind intensity analysis. J. Meteor. Soc. Japan, 84, 259-276.

Saito, K., T. Fujita, Y. Yamada, J. Ishida, Y. Kumagai, K. Aranami, S. Ohmori, R. Nagasawa, S. Kumagai, C. Muroi, T. Kato, H. Eito, and Y. Yamazaki, 2006: The operational JMA nonhydrostatic mesoscale model. Mon. Wea. Rev., 134, 1266-1298.

Saito, K., J. Ishida, K. Aranami, T. Hara, T. Segawa, M. Narita, and Y. Honda, 2007: Nonhydrostatic atmospheric models and operational development at JMA. J. Meteor. Soc. Japan, 85B, 271-304.

Sasaki, H., K. Kurihara, I. Takayabu, and T. Uchiyama, 2008: Preliminary experiments of reproducing the 
present climate using the non-hydrostatic regional climate model. SOLA, 4, 25-28.

Satake, Y., M. Inatsu, M. Mori, and A. Hasegawa, 2013: Tropical cyclone tracking using a neighbor enclosed area tracking algorithm. Mon. Wea. Rev., 141, 35393555.

Shimura, T., N. Mori, and M. A. Hemer, 2017: Projection of tropical cyclone-generated extreme wave climate based on CMIP5 multi-model ensemble in the western North Pacific. Climate Dyn., 49, 1449-1462.

Sugi, M., A. Noda, and N. Sato, 2002: Influence of the global warming on tropical cyclone climatology: An experiment with the JMA global model. J. Meteor. Soc. Japan, 80, 249-272.

Tory, K. J., S. S. Chand, R. A. Dare, and J. L. McBride, 2013: The development and assessment of a model-, grid-, and basin-independent tropical cyclone detection scheme. J. Climate, 26, 5493-5507.

Tory, K. J., S. S. Chand, R. A. Dare, and J. L. McBride, 2013: An assessment of a model-, grid-, and basinindependent tropical cyclone detection scheme in selected CMIP3 global climate models. J. Climate,
26, 5508-5522.

Ullrich, P. A., and C. M. Zarzycki, 2017: TempestExtremes: A framework for scale-insensitive pointwise feature tracking on unstructured grids. Geosci. Model Dev., 10, 1069-1090.

Walsh, K. J. E., M. Fiorino, C. W. Landsea, and K. L. McInnes, 2007: Objectively determined resolution-dependent threshold criteria for the detection of tropical cyclones in climate models and reanalyses. J. Climate, 20, 2307-2314.

Watanabe, S., A. Murata, H. Sasaki, H. Kawase, and M. Nosaka, 2019: Projection of change in tropical cyclone precipitation over Japan under a future climate with a high-resolution regional climate model. $J$. Meteor. Soc. Japan, in revision.

Wilks, D. S., 2011: Statistical Methods in the Atmospheric Sciences. 3rd Edition. Academic Press, Oxford, UK, 704 pp.

Zarzycki, C. M., D. R. Thatcher, and C. Jablonowski, 2017: Objective tropical cyclone extratropical transition detection in high-resolution reanalysis and climate model data. J. Adv. Model. Earth Syst., 9, 130-148. 